\title{
Carboxylation of an Ansa-Zirconocene Dinitrogen Complex: Regiospecific Hydrazine Synthesis from $\mathrm{N}_{2}$ and $\mathrm{CO}_{2}$.
}

Donald J. Knobloch, Hannah E. Toomey and Paul J. Chirik*

Department of Chemistry and Chemical Biology, Baker Laboratory

Cornell University, Ithaca, NY 14853

\author{
pc92@cornell.edu
}

- Supporting Information - 


\section{Table of Contents}

$\begin{array}{ll}\text { Experimental Section } & \text { S3 }\end{array}$

Characterization of N, N'-Dicarboxylated Hydrazines $\quad$ S9

$\begin{array}{lr}\text { Selected NMR Spectra } & \text { S10 }\end{array}$

$\begin{array}{lr}\text { Full Reference 1 From Manuscript } & \text { S13 }\end{array}$

$\begin{array}{ll}\text { References } & \text { S13 }\end{array}$ 


\section{Experimental Details}

General Considerations. All air- and moisture-sensitive manipulations were carried out using standard high vacuum line, Schlenk or cannula techniques or in an M. Braun inert atmosphere drybox containing an atmosphere of purified nitrogen. The M. Braun drybox was equipped with a cold well designed for freezing samples in liquid nitrogen. Solvents for air- and moisture-sensitive manipulations were dried and deoxygenated using literature procedures. ${ }^{1}$ Toluene, benzene, pentane and heptane were further dried by distillation from "titanocene". ${ }^{2}$ Deuterated solvents for NMR spectroscopy were distilled from sodium metal under an atmosphere of argon and stored over $4 \AA$ molecular sieves. Argon and hydrogen gas were purchased from Airgas Incorporated and passed through a column containing manganese oxide on vermiculite and $4 \AA$ molecular sieves before admission to the high vacuum line. Carbon dioxide was also dried over $4 \AA$ molecular sieves before admission to the high vacuum line. Trimethylsilyl iodide was purchased from Acros and dried over $4 \AA$ molecular sieves prior to use. The hafnocene dinitrogen

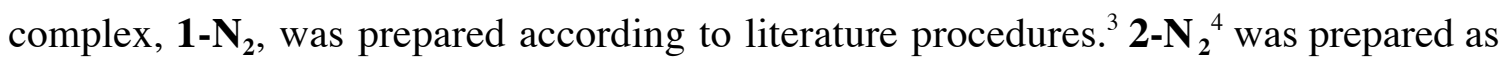
described previously using the diiodide precursor, $\mathbf{2}-\mathbf{I}_{\mathbf{2}}$.

${ }^{1} \mathrm{H}$ NMR spectra were recorded on a Varian Inova 400 Spectrometer operating at 399.860 MHz. All chemical shifts are reported relative to $\mathrm{SiMe}_{4}$ using ${ }^{1} \mathrm{H}$ (residual) chemical shifts of the solvent as a secondary standard. ${ }^{2} \mathrm{H},{ }^{13} \mathrm{C},{ }^{29} \mathrm{Si}$, and ${ }^{15} \mathrm{~N}$ NMR spectra were recorded on a Varian Inova 500 Spectrometer operating at 76.848, 125.716, 161.83, 99.320 and $50.663 \mathrm{MHz}$, respectively. ${ }^{2} \mathrm{H},{ }^{29} \mathrm{Si}$, and ${ }^{13} \mathrm{C}$ chemical shifts are reported relative to $\mathrm{SiMe}_{4}$ using chemical shifts of the solvent as a secondary standard where 
applicable. ${ }^{15} \mathrm{~N}$ chemical shifts are reported relative liquid to $\mathrm{NH}_{3}$ using an external standard.

Mass spectra were acquired using a JEOL GCMate II mass spectrometer operating at 500 (LRMS) resolving power (20\% FWHM) in positive ion mode and an electron ionization (EI) potential of $70 \mathrm{eV}$. Samples were introduced via a GC inlet using an Agilent HP 6890N GC equipped with a $30 \mathrm{~m}$ (0.25 m i.d.) HP-5ms capillary GC column. The carrier gas is helium with a flow rate of $1 \mathrm{~mL} / \mathrm{min}$. Samples were introduced into the GC using a split/splitless injector at $230{ }^{\circ} \mathrm{C}$ with a split ratio of $50: 1$. Infrared spectroscopy was conducted on a Mattson RS-10500 Research Series FT-IR spectrometer calibrated with a polystyrene standard. Elemental analyses were performed at Robertson Microlit Laboratories, Inc., in Madison, NJ.

Preparation of $\left[\mathrm{Me}_{2} \mathrm{Si}\left(\eta^{5}-\mathrm{C}_{5} \mathrm{Me}_{4}\right)\left(\eta^{5}-\mathrm{C}_{5} \mathrm{H}_{3}-3-{ }^{\mathrm{t}} \mathbf{B u}\right)\right] \mathrm{ZrI}_{2}\left(\mathbf{2}-\mathrm{I}_{2}\right)$. A $20 \mathrm{~mL}$ scintillation vial was charged with $1.31 \mathrm{~g}(0.0028 \mathrm{~mol})$ of $\left[\mathrm{Me}_{2} \mathrm{Si}\left(\eta^{5}-\mathrm{C}_{5} \mathrm{Me}_{4}\right)\left(\eta^{5}-\mathrm{C}_{5} \mathrm{H}_{3}-3-{ }^{\mathrm{t}} \mathrm{Bu}\right)\right] \mathrm{ZrCl}_{2}$ dissolved in approximately $10 \mathrm{~mL}$ of toluene. To the vial $4.55 \mathrm{~g}(0.023 \mathrm{~mol})$ of iodotrimethylsilane was added with stirring. The reaction was stirred at room temperature for 5 days. Excess iodotrimethylsilane and toluene were removed in vacuo, yielding a yellow solid identified as $\mathbf{2}-\mathbf{I}_{2}$ in quantitative yield. ${ }^{1} \mathrm{H}$ NMR (benzene- $d_{6}$ ): $\delta=0.25$ (s, 3H, Si $M e_{2}$ ), 0.33 (s, 3H, SiMe $e_{2}$ ), 1.57 (s, 9H, $\mathrm{C}_{5} \mathrm{H}_{3} \mathrm{CMe}$ ), 1.65 (s, 3H, $\mathrm{C}_{5} M e_{4}$ ), 2.28 (s, $\left.3 \mathrm{H}, \mathrm{C}_{5} M e_{4}\right), 2.34\left(\mathrm{~s}, 3 \mathrm{H}, \mathrm{C}_{5} M e_{4}\right), 5.26\left(\mathrm{~m}, 1 \mathrm{H}, \mathrm{C}_{5} H_{3} \mathrm{CMe}_{3}\right), 5.58\left(\mathrm{~m}, 1 \mathrm{H}, \mathrm{C}_{5} H_{3} \mathrm{CMe}_{3}\right), 7.45$ (m, $1 \mathrm{H}, \mathrm{C}_{5} H_{3} \mathrm{CMe}_{3}$ ). One $\mathrm{C}_{5} M e_{4}$ not observed. $\left\{{ }^{1} \mathrm{H}\right\}{ }^{13} \mathrm{C}$ NMR (benzene- $d_{6}$ ): $\delta=-0.94$ $\left(\mathrm{SiMe} e_{2}\right), 0.46(\mathrm{SiMe}), 15.91(\mathrm{Cp} M e), 16.58(\mathrm{Cp} M e), 16.92$ (CpMe), $18.35(\mathrm{Cp} M e), 31.78$ $\left(\mathrm{CMe}_{3}\right), 34.60\left(\mathrm{CMe}_{3}\right), 96.02,101.29,112.17,113.86,125.03,129.23,130.04,136.13$, $137.54,151.45(C p)$. 
Preparation of $\left(\left[\mathrm{Me}_{2} \mathrm{Si}\left(\eta^{5}-\mathrm{C}_{5} \mathrm{Me}_{4}\right)\left(\eta^{5}-\mathrm{C}_{5} \mathrm{H}_{3}-3-{ }^{t} \mathrm{Bu}\right)\right] \mathrm{Zr}\right)_{2}\left(\mathrm{~N}_{2} \mathrm{C}_{2} \mathrm{O}_{4}\right) \quad\left(2-\left(\mathrm{NCO}_{2}\right)_{2}\right)$. A J. Young NMR tube was charged with $0.045 \mathrm{~g}(0.056 \mathrm{mmol})$ of $\mathbf{2}-\mathbf{N}_{\mathbf{2}}$ and approximately 1 $\mathrm{mL}$ of pentane was added. On a high-vacuum line, two equivalents $(0.11 \mathrm{mmol})$ of $\mathrm{CO}_{2}$ were admitted to the tube via a calibrated gas bulb. The green color of the starting dinitrogen compound disappeared and an orange precipitate formed over several minutes. The tube was transferred to the dry box and the orange solid was collected by filtration, washed with cold pentane and dried in vacuo to obtain $2-\left(\mathbf{N C O}_{2}\right)_{2}$ in $76 \%$ yield. Anal. Calcd for $\mathrm{C}_{42} \mathrm{H}_{60} \mathrm{O}_{4} \mathrm{~N}_{2} \mathrm{Si}_{2} \mathrm{Zr}_{2}$ : C, 56.32; H, 6.75; N, 3.13. Found: C, 55.97; H, 6.94; N, 2.99. ${ }^{1} \mathrm{H}$ NMR (benzene- $d_{6}$ ): $\delta=0.48$ (s, 3H, SiMe $e_{2}$ ), 0.61 (s, 3H, SiMe $), 1.39$ (s, 9H, $\left.\mathrm{C}_{5} \mathrm{H}_{3} \mathrm{CMe} e_{3}\right), 1.91\left(\mathrm{~s}, 3 \mathrm{H}, \mathrm{C}_{5} M e_{4}\right), 1.99\left(\mathrm{~s}, 3 \mathrm{H}, \mathrm{C}_{5} M e_{4}\right), 2.10\left(\mathrm{~s}, 3 \mathrm{H}, \mathrm{C}_{5} M e_{4}\right), 2.19$ (s, 3H, $\left.\mathrm{C}_{5} M e_{4}\right), 5.79\left(\mathrm{~m}, 1 \mathrm{H}, \mathrm{C}_{5} H_{3} \mathrm{CMe}_{3}\right), 5.91\left(\mathrm{~m}, 1 \mathrm{H}, \mathrm{C}_{5} H_{3} \mathrm{CMe}_{3}\right), 6.76\left(\mathrm{~m}, 1 \mathrm{H}, \mathrm{C}_{5} H_{3} \mathrm{CMe}_{3}\right)$. $\left\{{ }^{1} \mathrm{H}\right\}{ }^{13} \mathrm{C}$ NMR (benzene- $\left.d_{6}\right): \delta=165.6\left(\mathrm{NCO}_{2}\right) .{ }^{15} \mathrm{~N}$ NMR (benzene- $\left.d_{6}\right): \delta=209.5\left({ }^{15} \mathrm{~N}_{2}\right)$. $\left({ }^{1} \mathrm{~J}_{\mathrm{CN}}=8.4 \mathrm{~Hz},{ }^{2} \mathrm{~J}_{\mathrm{CN}}=2.2 \mathrm{~Hz},{ }^{1} \mathrm{~J}_{\mathrm{NN}}=17.3 \mathrm{~Hz},{ }^{3} \mathrm{~J}_{\mathrm{CC}}=3.8 \mathrm{~Hz}\right) . \mathrm{IR}(\mathrm{KBr}): v=1481,1624 \mathrm{~cm}^{-1}$ $(\mathrm{C}=\mathrm{O}) ; v=1437,1581 \mathrm{~cm}^{-1}\left({ }^{13} \mathrm{C}=\mathrm{O}\right)$.

Preparation of $\left[\left(\mathrm{Me}_{3} \mathrm{Si}\right)\left(\mathrm{Me}_{3} \mathrm{SiO}_{2} \mathrm{C}\right) \mathrm{N}_{2}(3)\right.$. A J. Young NMR tube was charged with $0.010 \mathrm{~g}(0.011 \mathrm{mmol})$ of $\mathbf{2}-\left(\mathbf{N C O}_{2}\right)_{2}$ and approximately $0.5 \mathrm{~mL}$ benzene- $d_{6}$. To the solution was added an excess $(6.4 \mu \mathrm{L}(0.11 \mathrm{mmol}))$ of iodotrimethylsilane. The tube was shaken for several minutes, during which time the solution became yellow indicative of zirconium diiodide formation and hydrazine generation. A diastereomeric mixture of the product, 3, was characterized in situ as follows: ${ }^{1} \mathrm{H}$ NMR (benzene- $d_{6}$ ): $\delta=0.32,0.42$, $0.53\left(\left(\mathrm{CH}_{3}\right)_{3} \mathrm{SiN},\left(\mathrm{CH}_{3}\right)_{3} \mathrm{SiOCO}\right) .\left\{{ }^{1} \mathrm{H}\right\}{ }^{13} \mathrm{C}$ NMR (benzene- $\left.d_{6}\right): \delta 157.2,170.1 \mathrm{ppm}$ $\left(\mathrm{N}^{13} \mathrm{COSi}\right) ;\left({ }^{1} \mathrm{~J}_{\mathrm{CN}}=16.5 \mathrm{~Hz}\right)$. LRMS (m/z) [(Me $\left.\mathrm{Si}_{3}\left(\mathrm{Me}_{3} \mathrm{SiO}_{2} \mathrm{C}\right) \mathrm{N}\right]_{2}: 408,393,147,73$; $\left[\left(\mathrm{Me}_{3} \mathrm{Si}\right)\left(\mathrm{Me}_{3} \mathrm{SiO}_{2}{ }^{13} \mathrm{C}\right) \mathrm{N}\right]_{2}: 410,395,147,73 .\left[\left(\mathrm{Me}_{3} \mathrm{Si}\right)\left(\mathrm{Me}_{3} \mathrm{SiO}_{2}{ }^{13} \mathrm{C}\right){ }^{15} \mathrm{~N}\right]_{2}: 412,398,147$, 


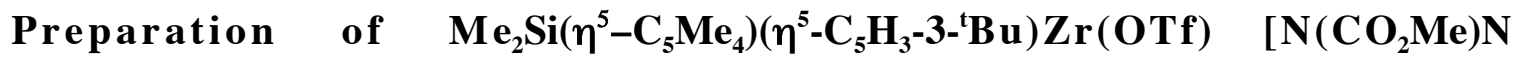
$\left.\left(\mathrm{CO}_{2} \mathbf{M e}\right)(\mathrm{Me})\right]$ (4). A $25 \mathrm{~mL}$ round bottom flask was charged with $0.080 \mathrm{~g}(0.089 \mathrm{mmol})$ 2-( $\left(\mathrm{NCO}_{2}\right)_{2}$ and slurried in approximately $10 \mathrm{~mL}$ of pentane. On a high vacuum line, the flask assembly was submerged in liquid nitrogen and degassed. At this temperature, 132 torr $(0.71 \mathrm{mmol})$ of methyl triflate were admitted. Upon thawing, a yellow solution formed after several minutes and was identified as an equimolar mixture of $\mathbf{4}$ and the corresponding bis(triflate) species, 2-(OTf) ${ }_{2}$. Excess methyl triflate and solvent were removed in vacuo and the remaining yellow solid was extracted into approximately $5 \mathrm{~mL}$ pentane and recrystallized at $-35{ }^{\circ} \mathrm{C}\left(45.1 \%\right.$ yield). ${ }^{1} \mathrm{H}$ NMR (benzene- $\left.d_{\sigma}\right): \delta 0.50(\mathrm{~s}, 3 \mathrm{H}$, $\mathrm{Si} M e_{2}$ ), 0.51 (s, 3H, SiMe $), 1.03$ (s, 9H, $\mathrm{C}_{5} \mathrm{H}_{3} \mathrm{CMe}_{3}$ ), 1.81 (s, 3H, $\mathrm{C}_{5} M e_{4}$ ), 2.03 (s, 3H, $\left.\mathrm{C}_{5} M e_{4}\right), 2.12\left(\mathrm{~s}, 3 \mathrm{H}, \mathrm{C}_{5} M e_{4}\right), 2.19\left(\mathrm{~s}, 3 \mathrm{H}, \mathrm{C}_{5} M e_{4}\right), 3.24$ (s, 3H, $\mathrm{CH}_{3} \mathrm{O}_{2} \mathrm{CNZr}$ ), 3.34 (s, $3 \mathrm{H}$, $\left.\mathrm{CH}_{3} \mathrm{NNZr}\right), 3.47\left(\mathrm{~s}, 3 \mathrm{H}, \mathrm{CH}_{3} \mathrm{O}_{2} \mathrm{CNNZr}\right), 5.33\left(\mathrm{~m}, 1 \mathrm{H}, \mathrm{C}_{5} H_{3} \mathrm{CMe}_{3}\right), 6.37(\mathrm{~m}, 1 \mathrm{H}$, $\mathrm{C}_{5} \mathrm{H}_{3} \mathrm{CMe}_{3}$ ), $7.22\left(\mathrm{~m}, 1 \mathrm{H}, \mathrm{C}_{5} \mathrm{H}_{3} \mathrm{CMe}_{3}\right) .\left\{{ }^{1} \mathrm{H}\right\}{ }^{13} \mathrm{C}$ NMR (benzene- $\left.d_{6}\right): \delta 38.26\left(\mathbb{N}^{13} \mathrm{CH}_{3}\right.$, $\left.{ }^{1} \mathrm{~J}_{\mathrm{CN}}=11.5 \mathrm{~Hz},{ }^{2} \mathrm{~J}_{\mathrm{CN}}=1.3 \mathrm{~Hz},{ }^{3} \mathrm{~J}_{\mathrm{CC}}=6.3 \mathrm{~Hz}\right), 53.40\left(\mathrm{NCO}_{2}{ }^{13} \mathrm{CH}_{3},{ }^{3} \mathrm{~J}_{\mathrm{CC}}=8.1 \mathrm{~Hz}\right), 52.91$ $\left(\mathrm{NCO}_{2}{ }^{13} \mathrm{CH}_{3},{ }^{3} \mathrm{~J}_{\mathrm{CC}}=4.5 \mathrm{~Hz}\right), 155.20\left(\mathrm{~N}^{13} \mathrm{CO}_{2},{ }^{1} \mathrm{~J}_{\mathrm{CN}}=29.4 \mathrm{~Hz}\right), 162.61\left(\mathrm{~N}^{13} \mathrm{CO}_{2},{ }^{1} \mathrm{~J}_{\mathrm{CN}}=10.4\right.$ Hz). IR (KBr): $v=1518 \mathrm{~cm}^{-1}, 1720(\mathrm{C}=\mathrm{O}) ; 1489,1678\left({ }^{13} \mathrm{C}=\mathrm{O}\right) ; 1014,1364\left(\mathrm{SO}_{3}\right) ; 1202$ $\left(\mathrm{CF}_{3}\right)$.

Preparation of $\mathrm{Me}_{2} \mathrm{Si}\left(\eta^{5}-\mathrm{C}_{5} \mathrm{Me}_{4}\right)\left(\eta^{5}-\mathrm{C}_{5} \mathrm{H}_{3}-3-{ }^{\mathrm{t}} \mathrm{Bu}\right) \mathrm{Zr}(\mathrm{OTf})_{2}$, (2-(OTf $\left.)_{2}\right)$. 2-(OTf $)_{2}$ was prepared as a byproduct of the reaction yielding 4.4 and 2-(OTf) $)_{2}$ were formed in an equimolar ratio. 2-(OTf) $)_{2}$ was independently prepared by addition of 2.2 equivalents of 
silver triflate $(0.012 \mathrm{~g}, 0.047 \mathrm{mmol})$ to a benzene solution containing $0.014 \mathrm{~g}(0.022$ mmol) of $\mathbf{2}-\mathbf{I}_{2}$. The AgI byproduct was removed via filtration and the solvent was

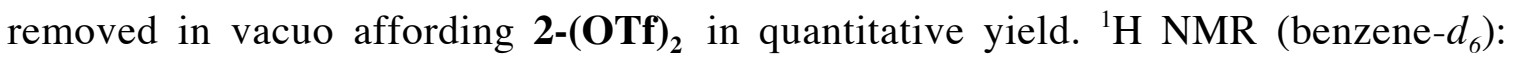
$\delta=0.30\left(\mathrm{~s}, 3 \mathrm{H}, \mathrm{Si} M e_{2}\right), 0.39$ (s, 3H, SiMe $), 1.09$ (s, 9H, $\left.\mathrm{C}_{5} \mathrm{H}_{3} \mathrm{CM} e_{3}\right), 1.62\left(\mathrm{~s}, 3 \mathrm{H}, \mathrm{C}_{5} M e_{4}\right)$, $1.68\left(\mathrm{~s}, 3 \mathrm{H}, \mathrm{C}_{5} M e_{4}\right), 1.94\left(\mathrm{~s}, 3 \mathrm{H}, \mathrm{C}_{5} M e_{4}\right), 2.00\left(\mathrm{~s}, 3 \mathrm{H}, \mathrm{C}_{5} M e_{4}\right), 5.48\left(\mathrm{~m}, 1 \mathrm{H}, \mathrm{C}_{5} H_{3} \mathrm{CMe}_{3}\right)$, $6.02\left(\mathrm{~m}, 1 \mathrm{H}, \mathrm{C}_{5} H_{3} \mathrm{CMe}_{3}\right), 7.11\left(\mathrm{~m}, 1 \mathrm{H}, \mathrm{C}_{5} H_{3} \mathrm{CMe}_{3}\right) .\left\{{ }^{1} \mathrm{H}\right\}{ }^{13} \mathrm{C}$ NMR (benzene- $\left.d_{6}\right): \delta=-$ $1.61\left(\mathrm{Si}^{2} e_{2}\right), 0.19$ (SiMe $), 10.97$ (CpMe), 12.75 (CpMe), 15.02 (CpMe), 15.17 (CpMe), $30.26\left(\mathrm{CMe}_{3}\right), 31.44\left(\mathrm{CF}_{3}\right), 31.76\left(\mathrm{CF}_{3}\right), 33.89\left(\mathrm{CMe}_{3}\right), 103.14,110.80,113.07,120.33$, $121.75,125.99,135.77,137.52,144.81,162.49(C p) .{ }^{19} \mathrm{~F}$ NMR (benzene- $\left.d_{6}\right): \delta=-75.27$, $-74.10\left(\mathrm{CF}_{3}\right) . \mathrm{IR}(\mathrm{KBr}): v=997,1360 \mathrm{~cm}^{-1}\left(\mathrm{SO}_{3}\right) ; 1198 \mathrm{~cm}^{-1}\left(\mathrm{CF}_{3}\right)$.

Preparation of $(\mathrm{H})\left(\mathrm{CO}_{2} \mathrm{Me}\right) \mathrm{NN}\left(\mathrm{CO}_{2} \mathrm{Me}\right)(\mathrm{Me})(5)$. The free hydrazine 5 was prepared by treating a benzene- $d_{6}$ solution of $4(0.005 \mathrm{~g}, 0.0078 \mathrm{mmol})$ with approximately $50 \mu \mathrm{L}$ of $\mathrm{H}_{2} \mathrm{O}$ and shaking for 20 minutes. The resulting mixture was filtered through a thin layer of celite and solvent was removed from the filtrate in vacuo leaving a pale yellow oil identified as 5. ${ }^{1} \mathrm{H}$ NMR (benzene- $\left.d_{6}\right): \delta=1.09\left(\mathrm{~s}, 3 \mathrm{H}, \mathrm{N} M e,{ }^{1} \mathrm{~J}_{\mathrm{HC}}=129.6 \mathrm{~Hz}\right.$ ), $3.31(\mathrm{br}$ s, $\left.3 \mathrm{H}, \mathrm{CO}_{2} M e,{ }^{1} \mathrm{~J}_{\mathrm{HC}}=146.3 \mathrm{~Hz}\right), 3.36\left(\mathrm{br} \mathrm{s}, 3 \mathrm{H}, \mathrm{CO}_{2} M e,{ }^{1} \mathrm{~J}_{\mathrm{HC}}=147.6 \mathrm{~Hz}\right)$, one N-H not observed. $\left\{{ }^{1} \mathrm{H}\right\}{ }^{13} \mathrm{C}$ NMR (benzene- $\left.d_{6}\right): \delta 31.01(\mathrm{~N} M e), 52.37\left(\mathrm{NCO}_{2} \mathrm{Me}\right), 53.00(\mathrm{Cp} M e)$, $156.03\left(\mathrm{CO}_{2} \mathrm{Me}\right), \quad 157.14\left(\mathrm{CO}_{2} \mathrm{Me}\right)$. GC-MS: LRMS (m/z) $(\mathrm{Me})\left(\mathrm{MeO}_{2} \mathrm{C}\right) \mathrm{N}-$ $\mathrm{N}\left(\mathrm{CO}_{2} \mathrm{Me}\right)(\mathrm{H}): 162,130,103,88,59 ;(\mathrm{Me})\left(\mathrm{MeO}_{2}{ }^{13} \mathrm{C}\right) \mathrm{N}-\mathrm{N}\left({ }^{13} \mathrm{CO}_{2} \mathrm{Me}\right)(\mathrm{H}): 164,132,104$, 89, 60; $\left(\mathrm{CD}_{3}\right)\left(\mathrm{CD}_{3} \mathrm{O}_{2} \mathrm{C}\right) \mathrm{N}-\mathrm{N}\left(\mathrm{CO}_{2} \mathrm{CD}_{3}\right)(\mathrm{H}): 171,136,109,94,62 ;\left(\mathrm{CD}_{3}\right)\left(\mathrm{CD}_{3} \mathrm{O}_{2}{ }^{13} \mathrm{C}\right) \mathrm{N}-$ $\mathrm{N}\left({ }^{13} \mathrm{CO}_{2} \mathrm{CD}_{3}\right)(\mathrm{H}): 173,138,110,95,63 ;\left({ }^{13} \mathrm{CH}_{3}\right)\left({ }^{13} \mathrm{CH}_{3} \mathrm{O}_{2} \mathrm{C}\right) \mathrm{N}-\mathrm{N}\left(\mathrm{CO}_{2}{ }^{13} \mathrm{CH}_{3}\right)(\mathrm{H}): 165,132$, $105,90,60$. 
Preparation of $\left(\mathrm{Me}_{3} \mathrm{Si}\right)\left(\mathrm{CO}_{2} \mathrm{Me}\right) \mathrm{NN}\left(\mathrm{CO}_{2} \mathrm{Me}\right)(\mathrm{Me})(6)$. The free hydrazine 6 was prepared by treating a benzene- $d_{6}$ solution of $4(0.006 \mathrm{~g}, 0.0089 \mathrm{mmol})$ with 10 equivalents of iodotrimethylsilane $(13 \mu \mathrm{L}, 0.089 \mathrm{mmol})$ at room temperature. Formation of 5 occurred within 5 minutes in $33.3 \%$ yield with concomitant formation of the corresponding zirconium diiodide compound, $\mathbf{2}-\mathbf{I}_{\mathbf{2}}$ and trimethylsilyl triflate. Characterization by ${ }^{1} \mathrm{H}$ NMR spectroscopy is consistent with previous literature values. ${ }^{5}$ ${ }^{1} \mathrm{H}$ NMR (dichloromethane- $d_{2}$ ): $\delta=0.25$ (s, 9H, SiMe $e_{3}$ ), 3.06 (s, 3H, NMe), 3.65 (s, 3H, $\left.\mathrm{CO}_{2} \mathrm{Me}\right), 3.70$ (s, 3H, $\mathrm{CO}_{2} \mathrm{Me}$ ). GC-MS: LRMS (m/z): 234, 219, 163, 130, 89, 73, 59. 


\section{N, N'-Dicarboxylated Hydrazine Characterization}<smiles>COC(=O)N(C(=O)O[Na])N([SiH3])[AsH3]</smiles>

From 1-NN $\left(\mathrm{CO}_{2}\right)_{2}$

Natural Abundance: $\mathrm{m} / \mathrm{z}=408$

${ }^{13} \mathrm{C}$ labeled: $\mathrm{m} / \mathrm{z}=410$

${ }^{13} \mathrm{C},{ }^{15} \mathrm{~N}$ labeled: $\mathrm{m} / \mathrm{z}=412$

${ }^{13} \mathrm{C}$ NMR, $\delta=157.7,157.23 \mathrm{ppm}$

${ }^{1} J(\mathrm{C}-\mathrm{N})=20.6 \mathrm{~Hz}$<smiles>COC(=O)N(C)N(C)C(=O)OC</smiles>

Natural Abundance: $\mathrm{m} / \mathrm{z}=162$

All $\mathrm{CD}_{3}$ groups: $\mathrm{m} / \mathrm{z}=171$

All $\mathrm{CD}_{3}$ groups, ${ }^{13} \mathrm{CO}_{2}: \mathrm{m} / \mathrm{z}=173$

All ${ }^{13} \mathrm{CH}_{3}$ groups: $\mathrm{m} / \mathrm{z}=165$<smiles>COC(=O)N([SiH3])N([SiH3])C(=O)OC</smiles>

3

Natural Abundance: $\mathrm{m} / \mathrm{z}=408$

${ }^{13} \mathrm{C}$ labeled: $\mathrm{m} / \mathrm{z}=410$

${ }^{13} \mathrm{C},{ }^{15} \mathrm{~N}$ labeled: $\mathrm{m} / \mathrm{z}=412$

${ }^{13} \mathrm{C}$ NMR, $\delta=157.2,170.1 \mathrm{ppm}$

${ }^{1} J(C-N)=16.5 \mathrm{~Hz}$<smiles>COC(=O)N(C)N(C)C(=O)OC</smiles>

Natural Abundance: $\mathrm{m} / \mathrm{z}=234$

Previously reported ${ }^{5}$

All data listed is experimental.

Figure S1. Characterization of various carboxylated hydrazines. 


\section{Selected NMR Spectra}

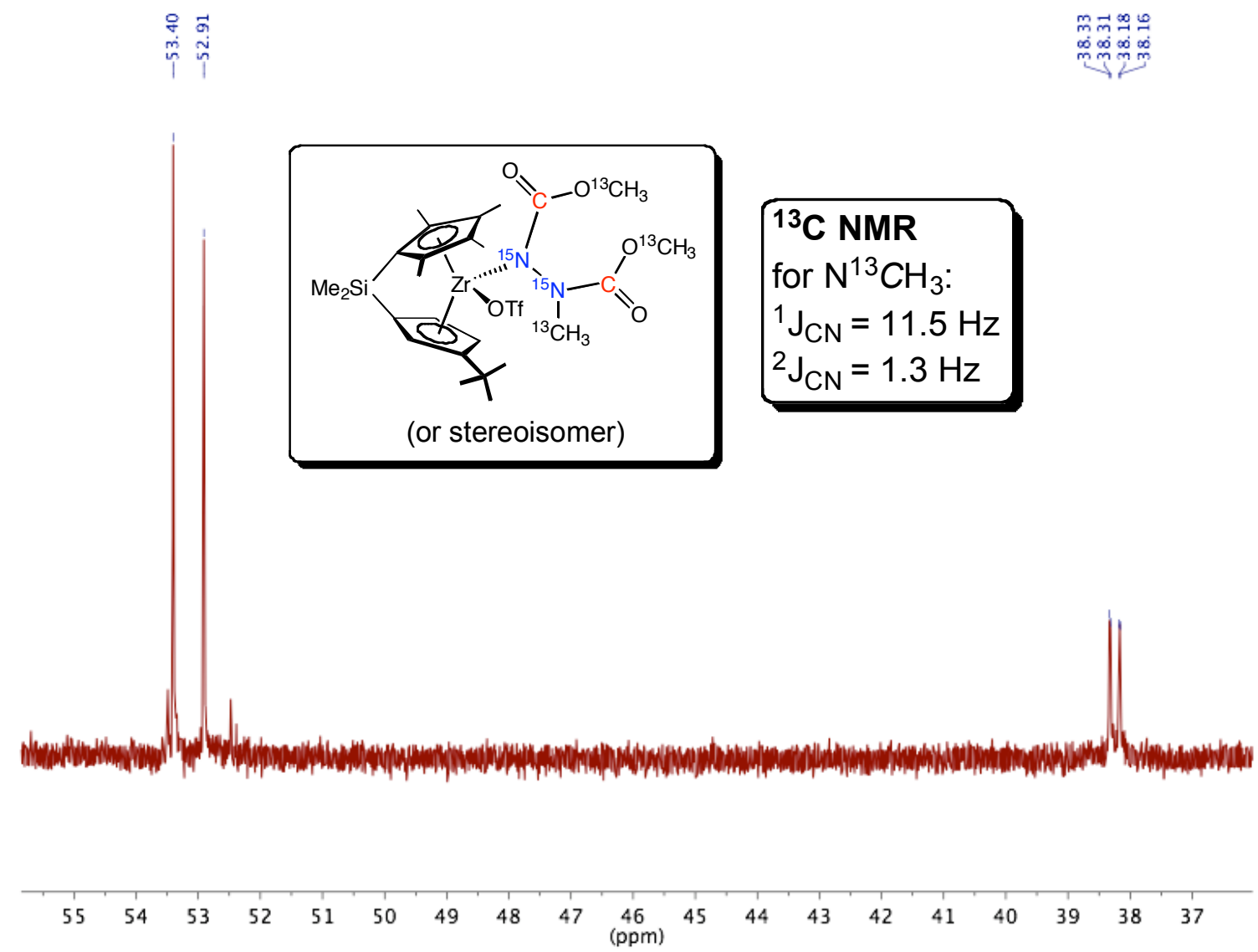

Figure S2. Partial ${ }^{13} \mathrm{C}$ NMR spectrum of $4\left({ }^{15} \mathrm{~N},{ }^{13} \mathrm{C}\right.$ labeled $)$.

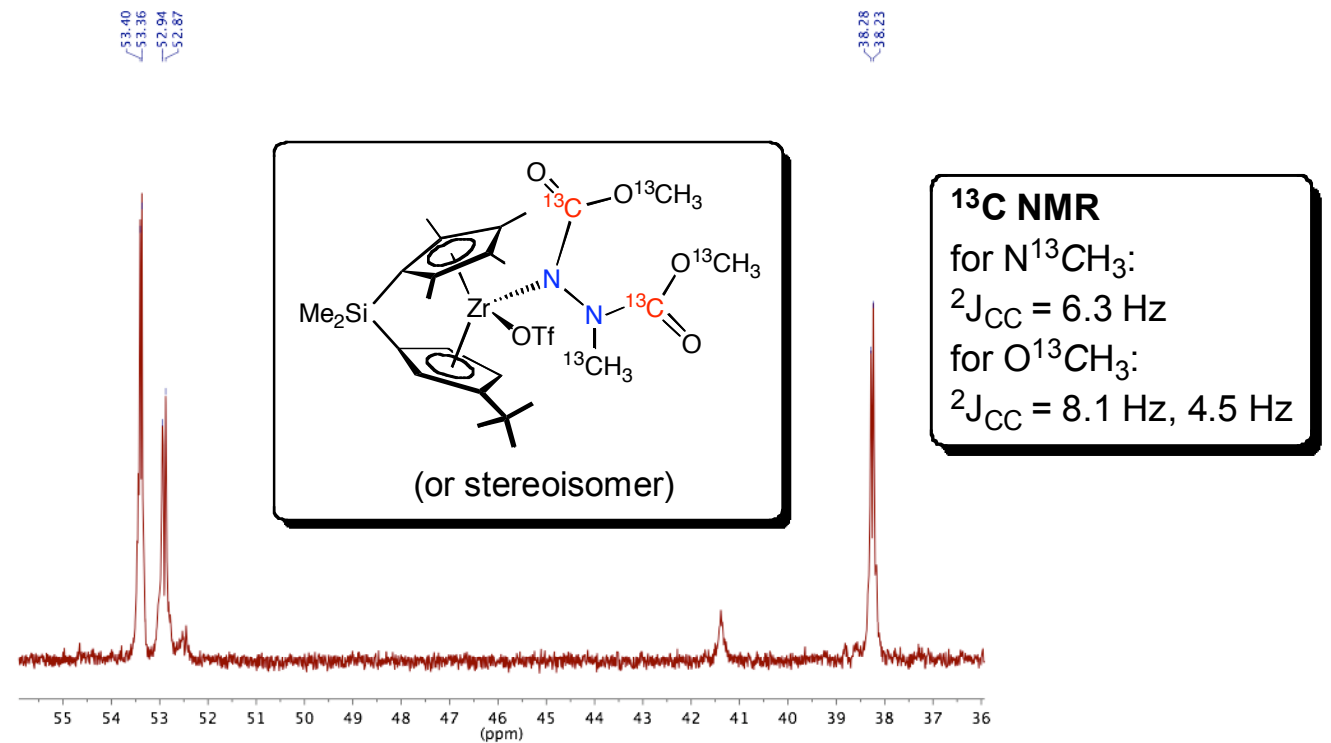

Figure S3. Partial ${ }^{13} \mathrm{C}$ NMR spectrum of $4\left({ }^{13} \mathrm{C}\right.$ labeled $)$. 


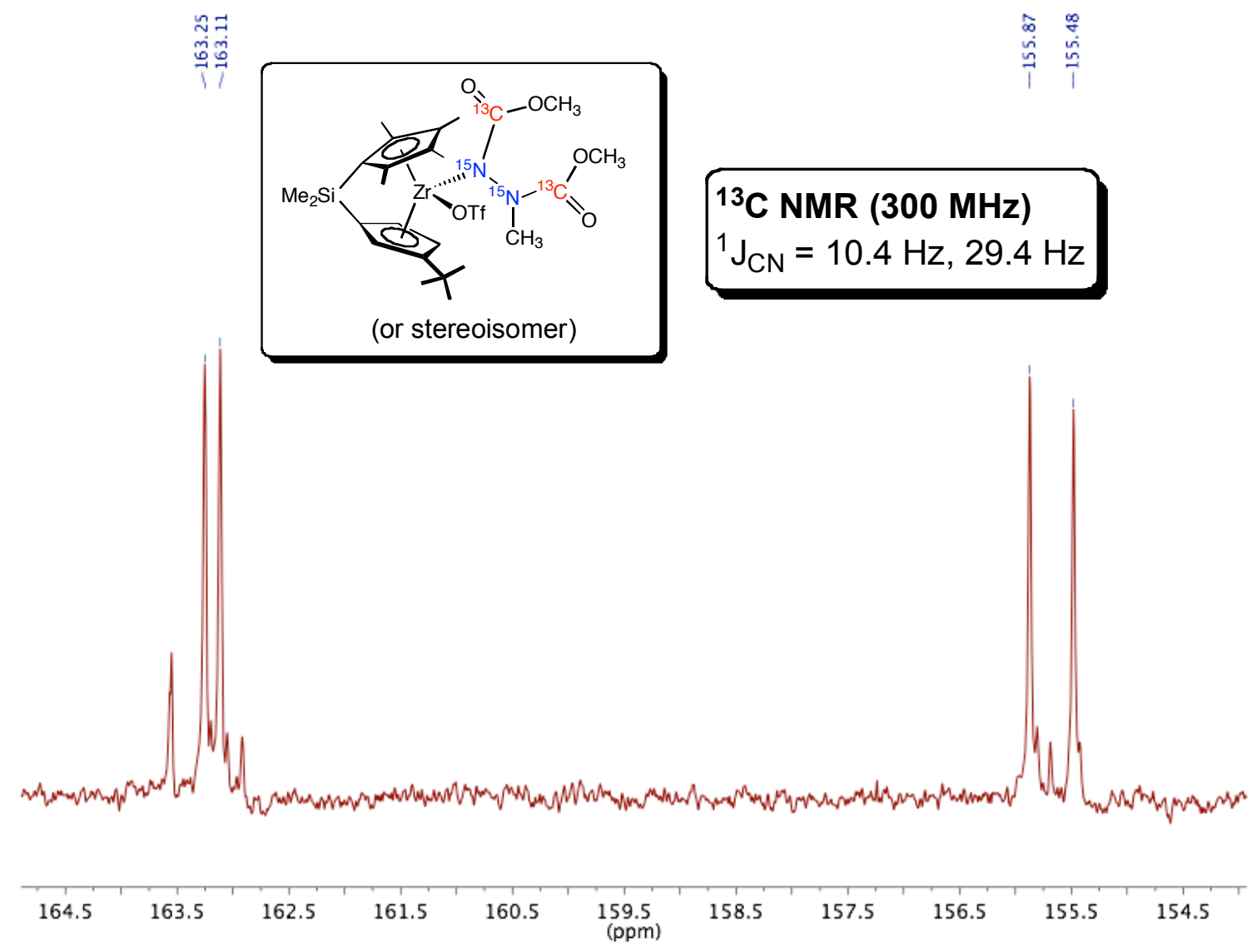

Figure S4. Partial ${ }^{13} \mathrm{C}$ NMR spectrum of $4\left({ }^{15} \mathrm{~N},{ }^{13} \mathrm{C}\right.$ labeled $)$. 


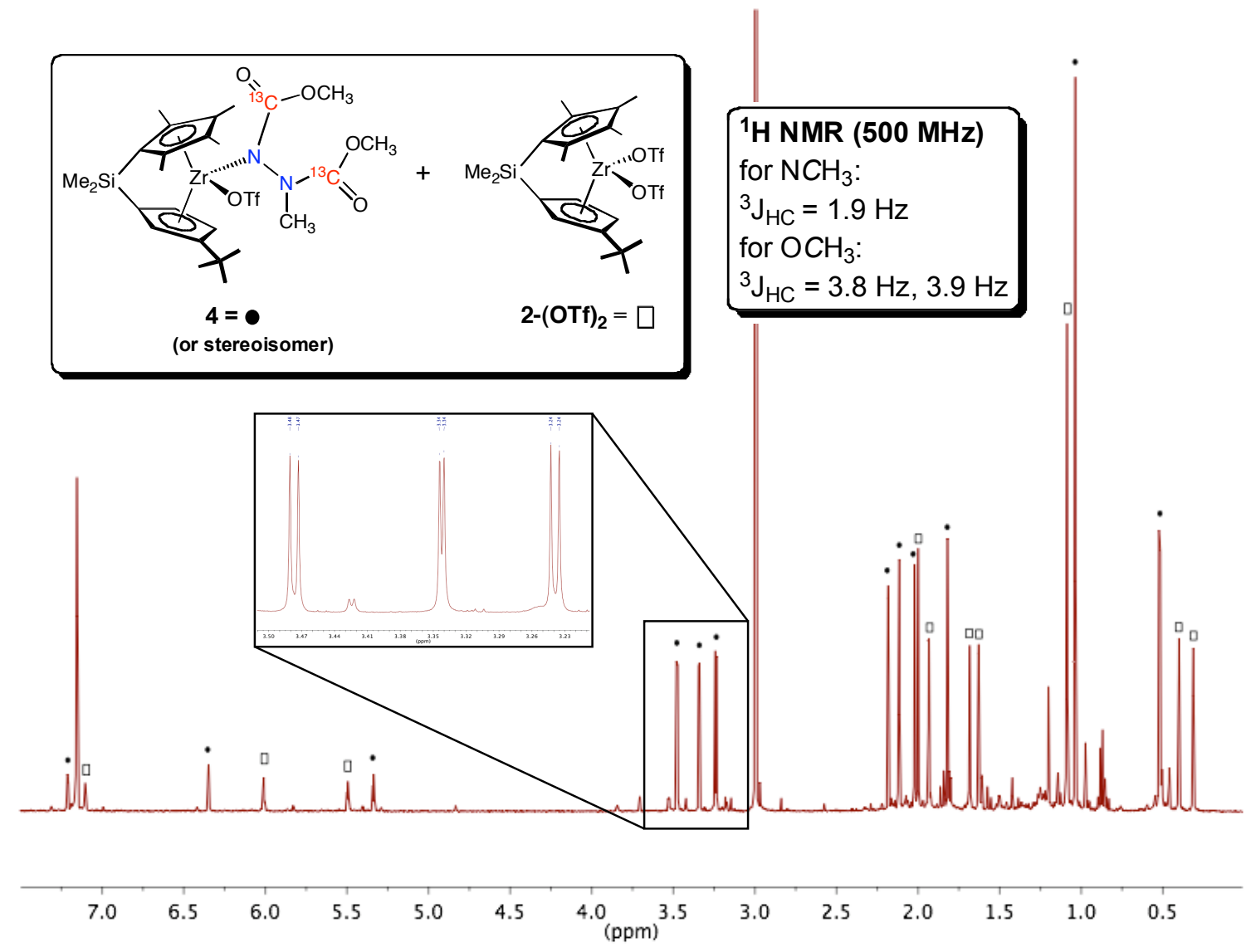

Figure S5. 'H NMR spectrum of 4 and 2-(OTf) $)_{2}\left({ }^{13} \mathrm{C}\right.$ labeled). 


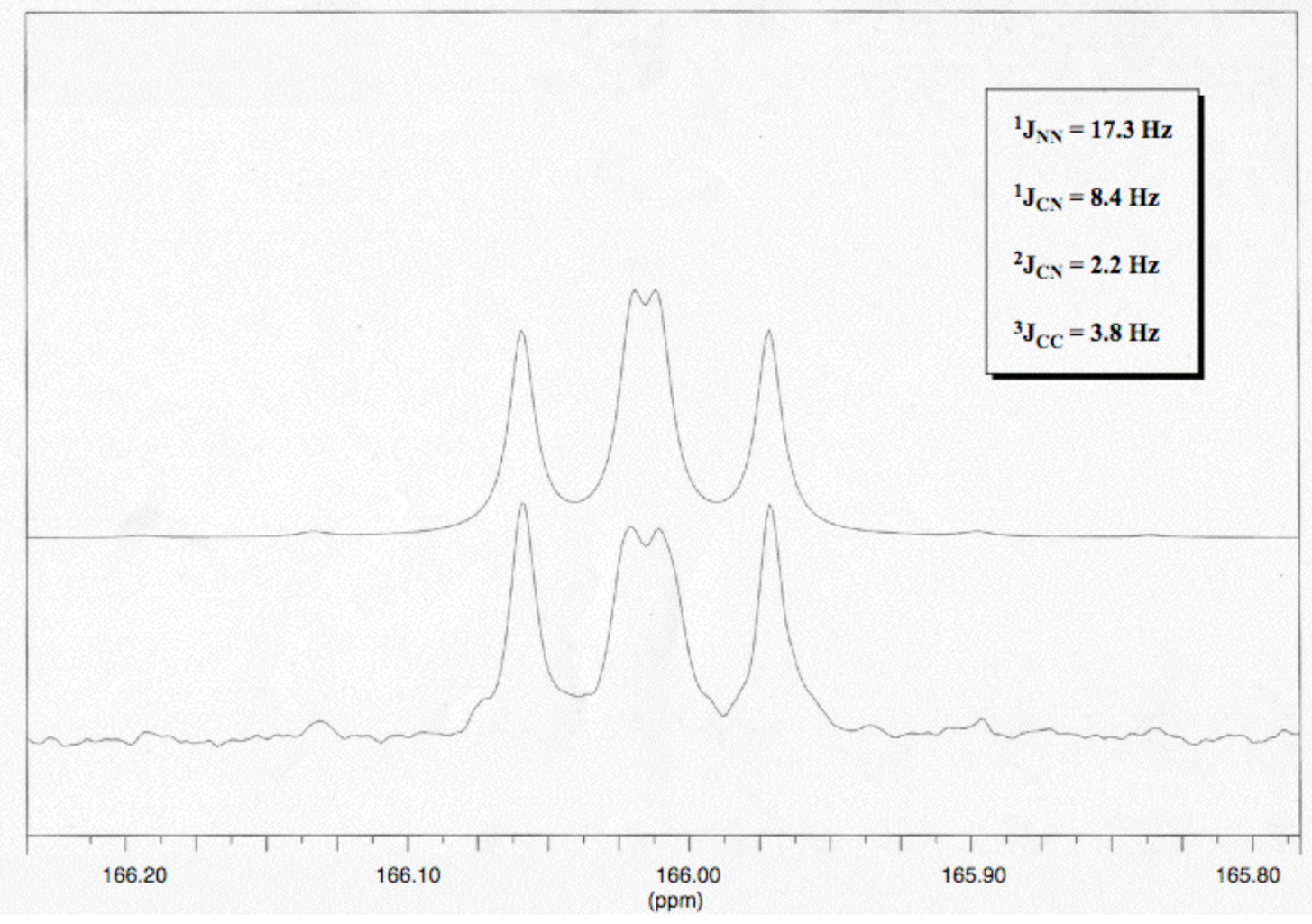

Figure S6. Partial experimental (bottom) and simulated (top) benzene- $d_{6}{ }^{13} \mathrm{C}$ NMR spectra of $2-\left(\mathbf{N}^{13} \mathrm{CO}_{2}\right)_{2}$.

\section{Full Reference 1 from Manuscript:}

Aresta, M.; Armor, J. N.; Barteau, M. A.; Beckman, E. J.; Bell, A. T.; Bercaw, J. E.; Creutz, C.; Dinjus, E.; Dixon, D. A.; Domen, K.; Dubois, D. L.; Eckert, J.; Fujita, E.; Gibson, D. H.; Goddard, W. A.; Goodman, D. W.; Keller, J.; Kubas, G. J.; Kung, H. H.; Lyons, J. E.; Manzer, L. E.; Marks, T. J.; Morokuma, K.; Nicholas, K. M.; Periana, R.; Que, L.; Rostrup-Nielson, J.; Sachtler, W. M. H.; Schmidt, L. D.; Sen, A.; Somorjai, G. A.; Stair, P. C.; Stults, B. R.; Tumas, W. Chem. Rev. 2001, 101, 953.

\section{References for Supporting Information.}

${ }^{1}$ Pangborn, A.B.; Giardello, M.A.; Grubbs, R.H.; Rosen, R.K.; Timmers, F.J. Organometallics 1996, 15, 1518. 
${ }^{2}$ Marvich, R.H.; Brintzinger, H.H. J. Am. Chem. Soc. 1971, 93, 2046.

${ }^{3}$ Bernskoetter, W. H.; Olmos, A. V.; Lobkovsky, E.; Chirik, P. J. Organometallics 2006, 25,1021 .

${ }^{4}$ Hanna, T. E.; Keresztes, I.; Lobkovsky, E.; Chirik, P. J. Inorg. Chem. 2007, 46, 1675.

${ }^{5}$ Kalinin, A.V.; Apasov, E.T. Bugaeva, S.V.; Ioffe, S.L.; Tartakovskii, V.A., Bulletin of the Academy of Sciences of the USSR Division of Chemical Science 1983, 32, 1282. 